\title{
Tanshinol upregulates the expression of aquaporin 5 in lung tissue of rats with sepsis
}

\author{
JIANFENG XU $\mathrm{X}^{1,2^{*}}$, LEI YANG ${ }^{1,3^{*}}$ and LIANG DONG ${ }^{1}$ \\ ${ }^{1}$ Department of Respiratory Medicine, Qilu Hospital of Shandong University, Jinan, Shandong 250012; \\ ${ }^{2}$ Department of Pulmonary Medicine, The Affiliated Yantai Yuhuangding Hospital of Qingdao University, Yantai, \\ Shandong 264000; ${ }^{3}$ Department of Tuberculosis, Shandong Provincial Chest Hospital, Jinan, Shandong 250032, P.R. China
}

Received November 22, 2017; Accepted June 15, 2018

DOI: $10.3892 / \mathrm{ol} .2018 .9026$

\begin{abstract}
Aquaporin 5 (AQP-5) is a water channel protein that is closely associated with non-small cell lung cancer tissues. The present study aimed to investigate the mechanism of tanshinol treatment on AQP-5 in the lung tissue of rats with sepsis. Animals in a rat sepsis model were randomly divided into six groups including blank control (ctrl), sham operation (SO), model (sepsis), low dose tanshinol (5 mg/kg/day; Tan-L), moderate dose tanshinol (10 mg/kg/day; Tan-M) and high dose tanshinol (20 mg/kg/day; Tan-H) groups. After 7 days of administration, the expression level of AQP-5 mRNA was detected by reverse transcription-quantitative polymerase chain reaction. The levels of interleukin-6 (IL-6) and tumor necrosis factor $\alpha(\mathrm{TNF}-\alpha)$ were measured by ELISA. Hematoxylin and eosin staining was used for histopathological observation. The expression levels of AQP-5, P38 and phosphorylated (P)-P38 protein in lung tissues were detected by western blot analysis. The expression levels of AQP-5 in the sepsis group were significantly decreased compared with those in ctrl and SO groups $(\mathrm{P}<0.01)$, while the levels of TNF- $\alpha$, IL-6 and p-P38 were significantly increased in sepsis group compared with those in ctrl and SO groups $(\mathrm{P}<0.01)$. Following tanshinol intervention, the expression levels of AQP-5 were significantly increased, while the levels of TNF- $\alpha$, IL- 6 and p-P38 were decreased compared with those in sepsis group. Tanshinol may upregulate the expression of AQP-5 by inhibiting the inflammatory cytokines and phosphorylation of P38, therefore protecting the lung tissue of rats with sepsis.
\end{abstract}

Correspondence to: Dr Liang Dong, Department of Respiratory Medicine, Qilu Hospital of Shandong University, 107 Wenhua West Road, Jinan, Shandong 250012, P.R. China

E-mail: gsy4471@163.com

*Contributed equally

Key words: aquaporins 5, lung tissue, tanshinol, sepsis, inflammatory response

\section{Introduction}

Sepsis is defined as a systemic inflammatory response syndrome with a demonstrated infectious etiology, of which the incidence is increasing (1-3). Severe sepsis may lead to organ dysfunction or tissue hypoperfusion (4), resulting in an unacceptably high mortality rate (5). Previous studies have established that the presently available therapeutics remain intractable and the mortality rate of sepsis remains high (5). A number of treatment methods, including low-dose hydrocortisone treatment and early goal-directed treatment, have been developed to increase the survival rate of the patients with sepsis $(6,7)$. However, corresponding observational studies have demonstrated that these aforementioned treatment methods failed to decrease mortality rates $(8,9)$. In addition, it was demonstrated that complications associated with the lungs (e.g., pneumonia, empyema, pleural effusion) often result in a high mortality rate in sepsis $(4,5)$. It would therefore be of great clinical value to identify novel drugs for the treatment of sepsis. It has previously been established that Salvia miltiorrhiza Bunge is widely used in Traditional Chinese Medicine for the treatment of cardiovascular disease, and tanshinol extracted from $S$. miltiorrhiza Bunge has been identified to be the primary active component (10). Tanshinol has now been widely applied in various human diseases including cancer (11), chronic kidney disease (12) and heart disease (13). Previous data have demonstrated that tanshinol may decrease oxidative stress to avoid deleterious effects on tissue (14). It also has been suggested that tanshinol may protect injured tissues resulting from various causes such as ischemia reperfusion and immune injury, by inhibiting the inflammatory response via different signaling pathways such as protein kinase $\mathrm{B} /$ nuclear factor $\mathrm{k}$ and macrophage-stimulating 1 /forkhead box $\mathrm{O} 3(15,16)$. Therefore, the antioxidant effects of tanshinol are promising (14). Furthermore, patients with sepsis suffer from severe oxidative stress (17). Consequently, it may be hypothesized that tanshinol may also exhibit protective roles in sepsis. However, the efficiency of tanshinol in sepsis, to the best of our knowledge, has not yet been described.

Aquaporin 5 (AQP-5) is a small water channel membrane protein that is associated with the major intrinsic protein such as p38 (18). Traditionally, AQP-5 is primarily expressed in a number of cell types, including large airway epithelia, 
acinar epithelial cells and type I alveolar epithelial cell apical membrane $(19,20)$. It was demonstrated that AQP-5 knockout may reduce the volume of liquid secreted from the upper airways, leading to the increased protein and salt concentration (21). Previous studies have demonstrated that AQP-5 may serve pivotal roles in a number of physiological processes and the development of various human diseases, including airway hyper-responsiveness, lung infection and acute lung injury (22-24). The expression level of AQP-5 is usually decreased following infection (23), and the deletion of AQP-5 may aggravate injury (25), indicating the important role of AQP-5 in the development of infection following injury. Direito et al (26) demonstrated that AQP-5 may be defined as a promising drug target and as a novel biomarker for cancer aggressiveness, with a high translational potential for therapeutics and diagnostics.

In the present study, the effects of tanshinol on the expression of AQP-5 were investigated and the possible mechanism was also explored.

\section{Materials and methods}

Animals. A total of 120 healthy male Sprague Dawley (SD) rats weighing from 200-230 g were purchased from Jinan Pengyue Experimental Animal Breeding Co., Ltd. (Jinan, China). All the rats were reared in specific-pathogen-free environment (22-25 $\mathrm{C}, 40-50 \%$ humidity, $12 \mathrm{~h}$ light and dark cycle) with access to food and water ad libitum. The permission for all animal experiments was obtained from the Animal Ethics Committee of Qilu Hospital of Shandong University (Jinan, China).

Sepsis model. The sepsis model was established by cecal ligation and puncture (CLP). Surgical anesthesia was performed by intraperitoneal (IP) injection of 3\% pentobarbibal (Jiangsu Hengrui Medicine Co., Ltd., Jiangsu, China) at a dose of $50 \mathrm{mg} / \mathrm{kg}$. A $3-\mathrm{cm}$ midline incision was made under sterile conditions; muscle layers were separated along the ventral white line and the peritoneum was cut to reach the abdominal cavity. The mesentery and cecum were separated and the cecum was ligated by surgical thread. The ligation end was punctured with a needle to release a small amount of stool. The intestine was then placed back, peritoneum and skin were sutured and the wound was disinfected.

Animal grouping. The experimental animals were randomly divided into six groups, including blank control (ctrl), sham operation (SO), model (sepsis), low dose tanshinol treatment (Tan-L), moderate dose tanshinol treatment (Tan-M) and high dose tanshinol treatment (Tan-H) groups. There were 20 rats in each group. Cecal ligation and puncture (CLP) was not performed in the ctrl group. Rats in the SO group were anesthetized, a $1 \mathrm{~cm}$ incision was made along the abdomen middle line; the cecum was then removed and returned after $2 \mathrm{~min}$ and the abdomen was then closed. The rats in Tans-L, Tans-M and Tans-H groups were treated once a day with intragastric administration using 5, 10 and $20 \mathrm{mg} / \mathrm{kg}$ tanshinol carboxymethyl cellulose-sodium (CMC-Na) suspension respectively, while $0.5 \%$ CMC-Na solution was used for rats in the remaining groups for 7 days. The mortality rate of the rats was observed and calculated every day for 7 days. The survival rate was expressed as a percentage compared to the ctrl group. Rats were anesthetized by IP injection of 3\% pentobarbibal at a dose of $50 \mathrm{mg} / \mathrm{kg}$ prior to the rats being sacrificed by cervical dislocation. Two sections of the lung tissues were collected from each animal; one was fixed in $10 \%$ buffered formalin at $4^{\circ} \mathrm{C}$ for $24 \mathrm{~h}$, and the other was maintained at $-80^{\circ} \mathrm{C}$.

$R T-q P C R$. Total RNA was extracted from lung tissue by TRIzol $^{\circledR}$ (Life Technologies; Thermo Fisher Scientific, Inc., Waltham, MA, USA) according to the manufacturer's protocol. Reverse transcription was performed using PrimeScript RT reagent kit $\left(42^{\circ} \mathrm{C}\right.$ for $15 \mathrm{~min}, 85^{\circ} \mathrm{C}$ for $5 \mathrm{sec}$ ) with DNA Eraser (Takara Biotechnology, Co. Ltd., Dalian, China). The level of AQP-5 mRNA was detected by RT-PCR with $\beta$-actin as the endogenous control. PCR quantitative analysis was performed with CFX96 Real Time PCR detection system (Bio Rad Laboratories, Inc., Hercules, CA, USA) with SYBR green I dye detection kit (Takara Biotechnology, Co. Ltd.). The following primers were used: 5'-TGGGTCTTCTGGGTAGGGCCT ATTGT-3' (sense) and 5'-GCCGGCTTTGGCACTTGAGAT ACT-3' (anti-sense) for AQP-5; 5'-ATCATGTTTGAGACC TTCAACA-3' (sense) and 5'-CATCTCTTGCTCGAAGTC CA-3' (anti-sense) for $\beta$-actin. PCR was performed as follows: $94^{\circ} \mathrm{C}$ pre-denaturation for $1 \mathrm{~min}$, followed by 40 cycles of $94^{\circ} \mathrm{C}$ for $20 \mathrm{sec}, 55^{\circ} \mathrm{C}$ for $20 \mathrm{sec}$ and $72^{\circ} \mathrm{C}$ for $20 \mathrm{sec}$. Quantification was performed by normalizing the cycle threshold values to those of $\beta$-actin and analyzing results using the $2^{-\Delta \Delta C q}$ method (27). The results of the experiment are the average of the three repeated samples.

ELISA. The supernatants were collected from the rat lung tissue homogenate, and the respective TNF- $\alpha$ (catalog no. ELR-TNFa-CL-5) and IL-6 (catalog no. ELR-IL6-2) ELISA kits (RayBiotech Inc., Norcross, GA, USA) were used. Optical density values (OD values) were read using an ELISA plate scanner (KHB ST-360; Shanghai Danding Company, China) at $490 \mathrm{~nm}$. The results of the experiment are the average of the three repeated samples.

Hematoxylin and eosin staining. The fixed lung tissue samples were dehydrated, embedded in paraffin and sliced $(4 \mu \mathrm{m})$. Subsequent to dewaxing with xylene, hydration was performed using a series of graded concentrations of ethanol $(100 \%$ ethanol for $5 \mathrm{~min}, 95 \%$ ethanol for $1 \mathrm{~min}, 80 \%$ ethanol for $5 \mathrm{~min}, 75 \%$ ethanol for $5 \mathrm{~min}$ and distilled water for $2 \mathrm{~min}$ ) at room temperature. Then stained with hematoxylin and eosin at room temperature for $12 \mathrm{~min}$. The sections were observed with an optical microscope (magnification, x100).

Western blot analysis. A total of $0.2 \mathrm{~g}$ of lung tissue with $1.8 \mathrm{ml} 0.9 \%$ of saline was obtained, chopped, centrifuged at $5,000 \mathrm{x} \mathrm{g}$ for $10 \mathrm{~min}$ at $4^{\circ} \mathrm{C}$ and the supernatant of homogenized lung tissue was obtained. The concentration of protein was measured by bicinchoninic acid assay (BCA). Protein samples $(40 \mu \mathrm{g})$ were separated via 10\% SDS-PAGE and transferred to a polyvinylidene fluoride membrane. The membrane was washed and blocked with TBS containing Tween-20 (TBST) solution and 5\% skimmed milk powder. The primary antibodies against AQP-5 (1:1,200, catalog no. orb48020), 
Table I. Survival rates of the rats in each group.

Surviving rats (day)

\begin{tabular}{|c|c|c|c|c|c|c|c|c|c|}
\hline Groups & 0 & 1 & 2 & 3 & 4 & 5 & 6 & 7 & $\begin{array}{l}\text { Survival } \\
\text { rate (\%) }\end{array}$ \\
\hline Ctrl & 20 & 20 & 20 & 20 & 20 & 20 & 20 & 20 & 100 \\
\hline SO & 20 & 19 & 19 & 19 & 19 & 19 & 19 & 19 & 95.0 \\
\hline Sepsis & 20 & 11 & 7 & 6 & 5 & 5 & 5 & 5 & 25.0 \\
\hline Tan-L & 20 & 14 & 11 & 9 & 9 & 9 & 9 & 9 & 45.0 \\
\hline Tan-M & 20 & 16 & 14 & 13 & 12 & 12 & 12 & 12 & 60.0 \\
\hline Tan-H & 20 & 17 & 16 & 15 & 15 & 15 & 15 & 15 & 75.0 \\
\hline
\end{tabular}

Ctrl, control; SO, sham operation group; Sepsis, model group; Tan-L, low dose tanshinol group (5 mg/kg); Tan-M moderate dose tanshinol group $(10 \mathrm{mg} / \mathrm{kg})$; Tan-H, high dose tanshinol group $(20 \mathrm{mg} / \mathrm{kg}) . \mathrm{n}=20$ for all groups.

p-P38 (1:1,500, catalog no. orb128324), P38 (1:12,00, catalog no. orb338949) and $\beta$-actin (1:2,000, catalog no. orb178392) (all Biorbyt Ltd., Cambridge, UK) were diluted with TBST solution containing 3\% bovine serum albumin (catalog no. orb334844, Biorbyt Ltd., Cambridge, UK). The membrane was incubated with the primary antibodies at $4^{\circ} \mathrm{C}$ overnight. The membrane was washed with TBST, and the horseradish peroxidase-conjugated secondary antibody (1:5,000, catalog no. orb345943, Biorbyt Ltd.) was diluted with PBS and incubated with the membrane for $1 \mathrm{~h}$ at room temperature. Subsequent to washing with TBST, the membrane was exposed to X-ray films (Light Labs Inc., Dallas, TX, USA) for color development with electrochemiluminescence Prime Western Blotting Detection reagent (Thermo Fisher Scientific, Inc.). Image J 2.1 software (National Institutes of Health, Bethesda, MD, USA) was used to analyze the results. $\beta$-actin was used as an endogenous control. The results of the experiment are the average of the three repeated samples.

Statistical analysis. Statistical analysis was performed using SPSS version 19.0 (IBM Corp., Armonk, NY, USA) statistical software. All data are presented as mean \pm standard deviation. Multiple comparisons were evaluated by one-way analysis of variance followed by Dunnett's post-hoc test. $\mathrm{P}<0.05$ was considered to indicate a statistically significant difference.

\section{Results}

Survival rates of rats in each group. The mortalities of rats in each group are summarized in Table I. No mortalities in the ctrl group and only 1 mortality in the SO group were identified in all the 20 rats, while only 5 rats were left in sepsis group at the fourth day following surgery. Compared with the model group, the survival rates were increased in rats of Tan-L, Tan-M and Tan-H groups following treatment with the different doses of tanshinol, indicating that tanshinol treatment may significantly increase the survival rate of rats with sepsis.

Tanshinol upregulates the $m R N A$ expression of AQP-5 in rats with sepsis. The mRNA expression level of AQP-5 in the sepsis group was significantly decreased compared with that in the ctrl and SO groups $(\mathrm{P}<0.01)$. Compared with the sepsis

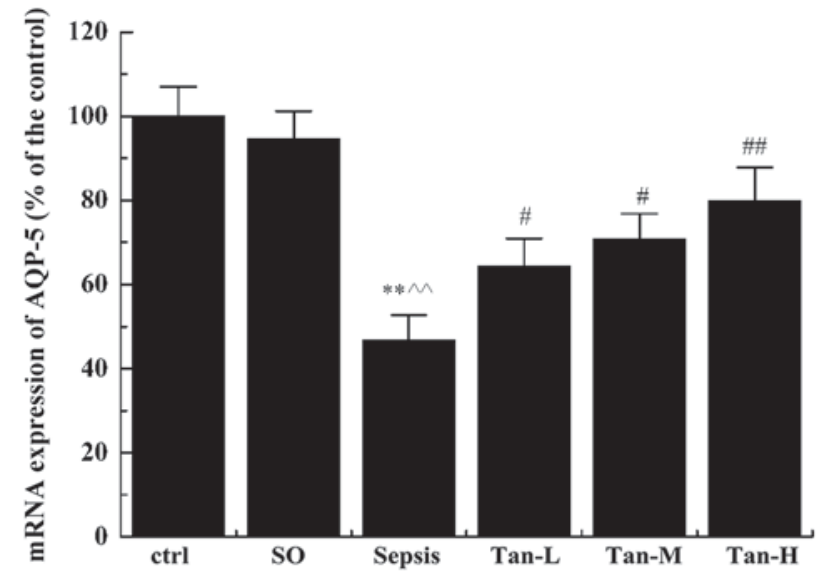

Figure 1. mRNA levels of AQP-5 in rats of each group. All data are presented as mean \pm standard deviation, ${ }^{* *} \mathrm{P}<0.01$ vs. ctrl group, ${ }^{\wedge} \mathrm{P}<0.01$ vs. SO group; ${ }^{\#} \mathrm{P}<0.05$ vs. Sepsis group; ${ }^{\# \#} \mathrm{P}<0.01$ vs. Sepsis group. AQP-5, Aquaporin 5; ctrl, control; SO, sham operation group; Sepsis, model group; Tan-L, low dose tanshinol group (5 mg/kg); Tan-M, moderate dose tanshinol group (10 mg/kg); Tan-H, high dose tanshinol group $(20 \mathrm{mg} / \mathrm{kg}) . \mathrm{n}=20$ for all groups.

group, the mRNA expression level of AQP-5 was significantly increased in a dose-dependent manner following treatment with tanshinol $(\mathrm{P}<0.05$; Fig. 1), indicating that treatment with tanshinol may increase the mRNA expression level of AQP-5.

Tanshinol decreases the levels of proinflammatory cytokines in rats with sepsis. Compared with the ctrl group, the levels of TNF- $\alpha$ and IL-6 in the sepsis group were significantly increased ( $\mathrm{P}<0.01$; Fig. 2). Compared with the sepsis group, the concentration of TNF- $\alpha$ and IL- 6 in rats with sepsis was significantly decreased following treatment with tanshinol in a dose-dependent manner. These data suggests that tanshinol may decrease the increased levels of TNF- $\alpha$ and IL- 6 caused by sepsis.

Tanshinol protects the lung tissue of rats with sepsis. As indicated in Fig. 3, the bronchial epithelium of the lung tissues in the ctrl and SO groups were intact. As indicated in Fig. 3A and B, it was without inflammatory cell infiltration, hemorrhage and edema in lung tissue. As shown 

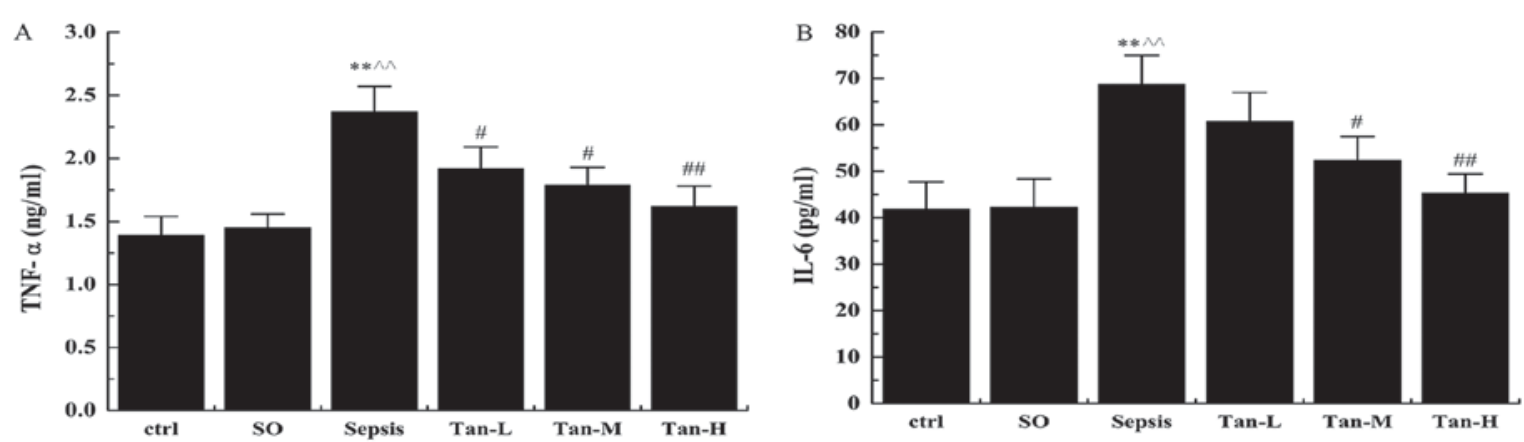

Figure 2. ELISA analysis the effects of tanshinol on the levels of proinflammatory cytokines in rats with sepsis. (A) TNF- $\alpha$ levels of lung tissue in rats of each group. (B) IL-6 levels of lung tissue in rats of each group. All the data are presented as mean \pm standard deviation, ${ }^{* * *} \mathrm{P}<0.01$ vs. ctrl group; ${ }^{\wedge} \mathrm{P}<0.01$ vs. SO group; ${ }^{~} \mathrm{P}<0.05$ vs. Sepsis group; ${ }^{\# \#} \mathrm{P}<0.01$ vs. with Sepsis group. TNF- $\alpha$, tumor necrosis factor- $\alpha$; IL-6, interleukin-6; ctrl, control; SO, sham operation group; Sepsis, model group; Tan-L, low dose tanshinol group (5 mg/kg); Tan-M, moderate dose tanshinol group (10 mg/kg); Tan-H, high dose tanshinol group $(20 \mathrm{mg} / \mathrm{kg}) . \mathrm{n}=20$ for all groups.

A

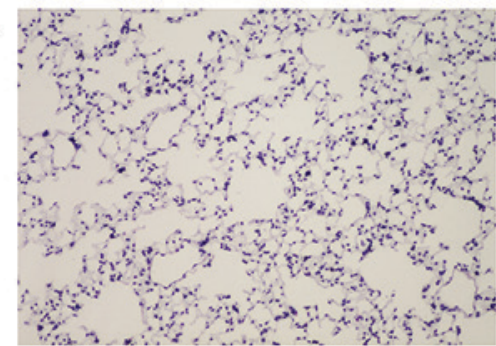

B

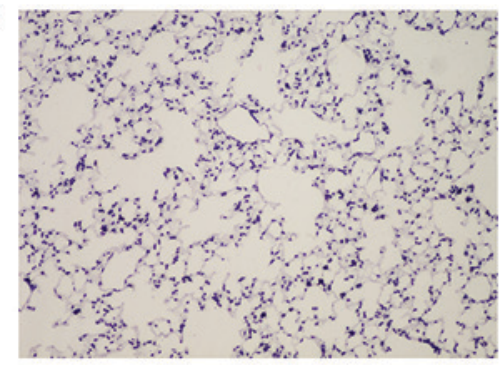

$\mathrm{C}$
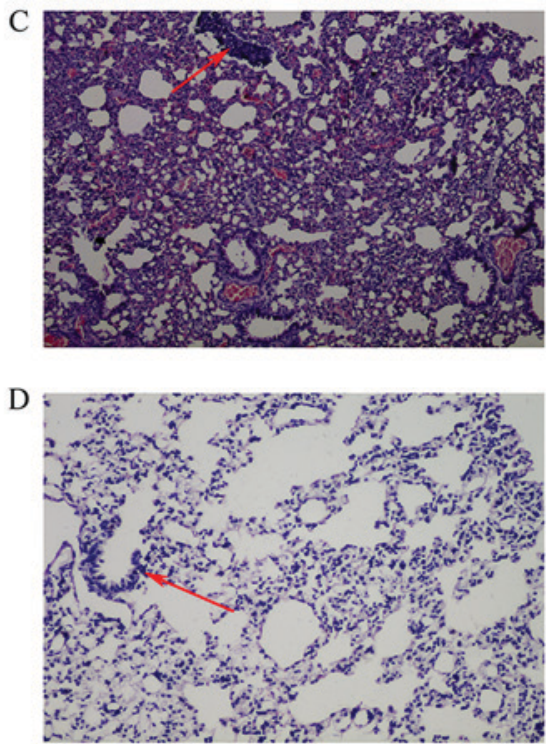

E

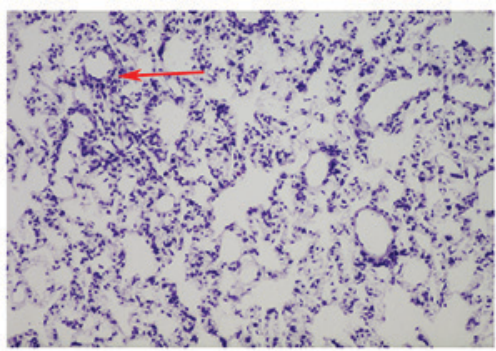

F

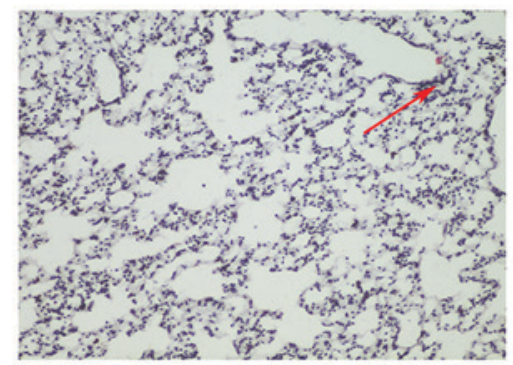

Figure 3. Pathological imaging of rat lung tissue (magnification, x100) following hematoxylin and eosin staining. (A) ctrl group; (B) SO group; (C) Sepsis group; (D) Tans-L group; (E) Tans-M group; (F) Tans-H group. Red arrows indicate inflammatory cell infiltration. ctrl, control; SO, sham operation group; Sepsis, model group; Tan-L, low dose tanshinol group ( $5 \mathrm{mg} / \mathrm{kg}$ ); Tan-M, moderate dose tanshinol group (10 mg/kg); Tan-H, high dose tanshinol group (20 mg/kg).

in Fig. 3C, hyperplasia of lung tissue, degeneration of some bronchial epithelium, infiltration of neutrophils in the lung and bronchus, and focal hemorrhage were identified in the rats of sepsis group. As shown in Fig. 3D-F, pulmonary interstitial hyperplasia, neutrophil infiltration in lung tissue and bronchus, bleeding and edema were improved significantly following treatment with tanshinol in dose-dependent manner.

Tanshinol regulates the expression of AQP-5, P38 and p-P38 in lung tissue of rats with sepsis. The expression of the AQP-5 protein was detected by western blot analysis. The expression of the AQP-5 protein was significantly inhibited in the sepsis group compared with that in the ctrl or the SO group $(\mathrm{P}<0.01)$, while the expression of AQP-5 protein was significantly increased compared with the sepsis group, following treatment with tanshinol in a dose-dependent manner (Fig. 4A). The phosphorylation level of P38 was also detected by western blot analysis. The phosphorylation of P38 in the lung tissue of rats in the sepsis group was significantly increased compared with that of the ctrl and SO groups $(\mathrm{P}<0.01$; Fig. $4 \mathrm{~B})$. Compared with the sepsis group, the P-P38:P38 ratio in the treatment with tanshinol groups was significantly decreased $(\mathrm{P}<0.05$; Fig. 4B). These data suggests that tanshinol may regulate the expression of AQP-5, P38 and p-P38 at the protein level.

\section{Discussion}

As the primary active component of the Traditional Chinese Medicine compound S. miltiorrhiza Bunge, tanshinol has been widely used in clinical practice for the treatment of various human diseases (for example, arteriosclerosis, myocardial ischemia) in order to provide a more favorable patient outcome (10-13). The present study verified that the efficiency of tanshinol on treatment with sepsis was confirmed with the establishment of rat sepsis model by CLP. It has been previously established that sepsis, which may 

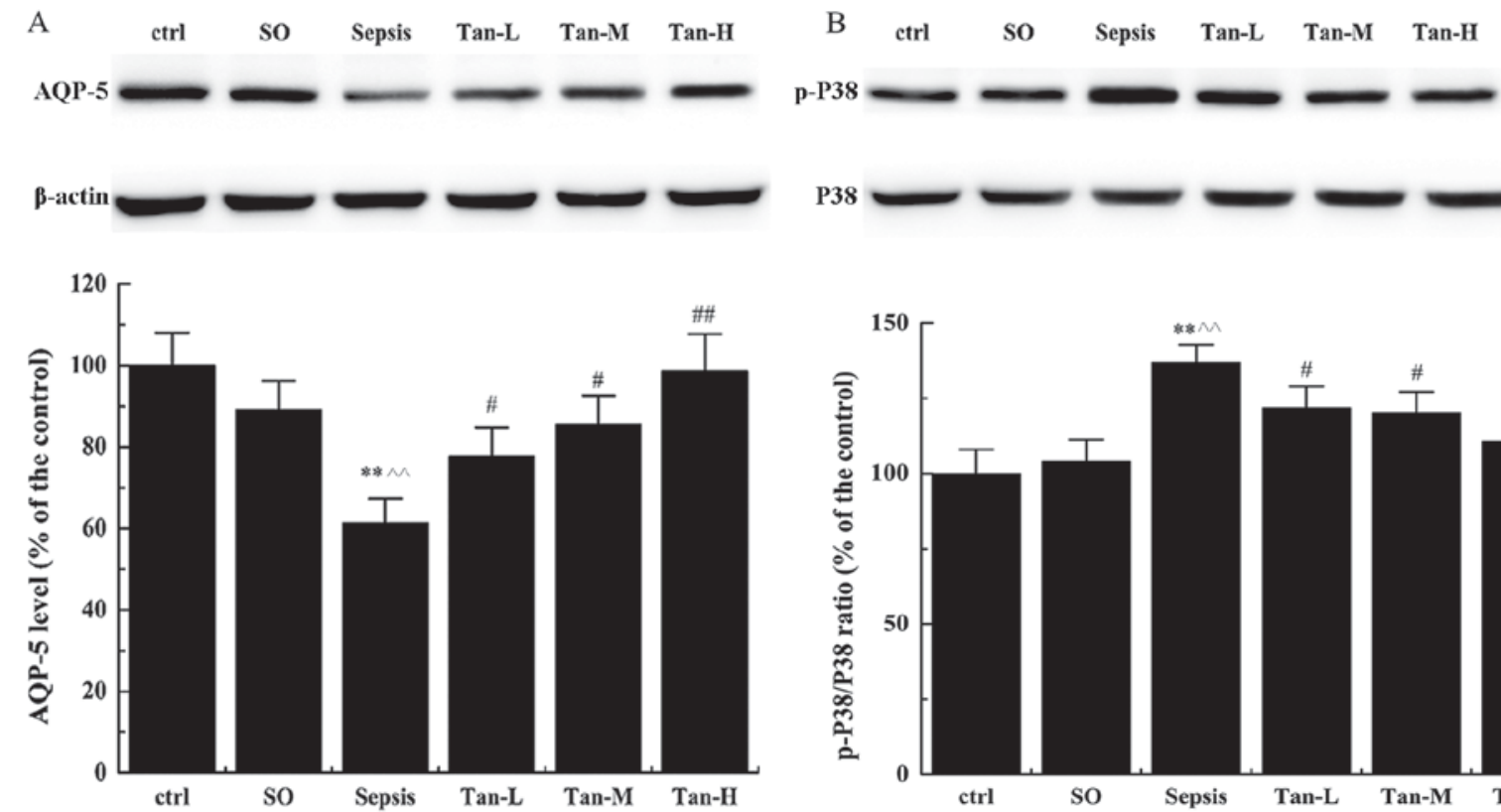

P38
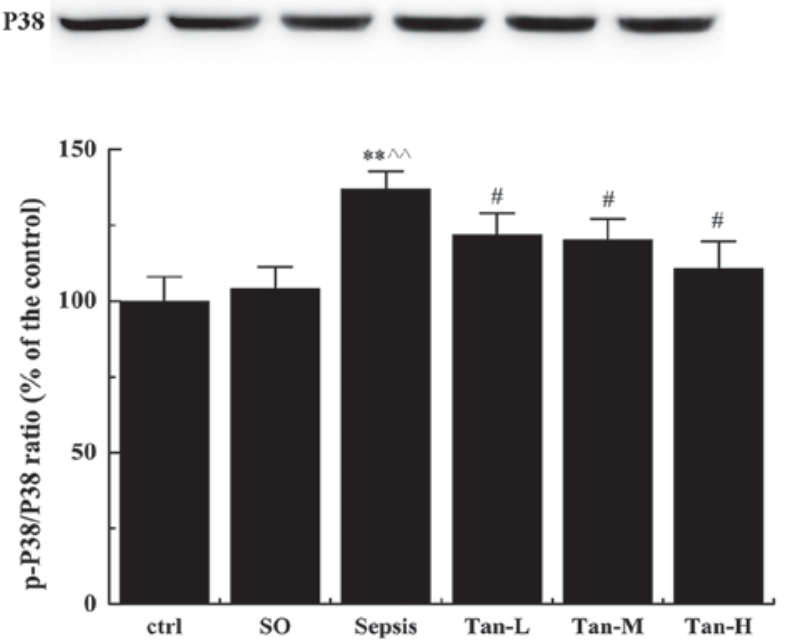

Figure 4. Effects of tanshinol on the expression of AQP-5, P38 and p-P38 in lung tissue of rats with Sepsis by western blot analysis. (A) Western blot and quantification of the expression levels of AQP-5 in rats of different group. (B) Western blot and quantification of the levels of P38 and p-P38 in rats of different group. All the data are presented as mean \pm standard deviation. ${ }^{* *} \mathrm{P}<0.01$ vs. ctrl group; ${ }^{\wedge} \mathrm{P}<0.01$ vs. SO group; ${ }^{\#} \mathrm{P}<0.05$ vs. Sepsis group; ${ }^{\# \#} \mathrm{P}<0.01 \mathrm{vs}$. Sepsis group. AQP-5, Aquaporin 5; P-, phosphorylated; SO, sham operation group; Sepsis, model group; Tan-L, low dose tanshinol group (5 mg/kg); Tan-M, moderate dose tanshinol group $(10 \mathrm{mg} / \mathrm{kg})$; Tan-H, high dose tanshinol group $(20 \mathrm{mg} / \mathrm{kg}) . \mathrm{n}=20$ for all groups.

lead to organ dysfunction or tissue hypoperfusion, is a major health problem due to its difficulty to cure and is strongly associated with high mortality and morbidity (5). Previously, a number of treatment modalities such as continuous blood purification (3) and physical therapy (28) have been used to increase the survival rate of patients with sepsis. However, the majority of treatment methods for sepsis have been demonstrated to be non-effective in decreasing the mortality rate by observational studies $(8,9)$. Therefore, early diagnoses and identification of efficient therapies for sepsis are integral to resolving these problems.

The results of the present study demonstrated that the application of tanshinol in the treatment of sepsis elevates the survival rates of rats. Furthermore, histopathological examination revealed that pulmonary interstitial hyperplasia, neutrophil infiltration of lung tissue and bronchus, bleeding and edema were improved significantly following treatment with tanshinol. These data suggested that tanshinol exerts high efficiency on the treatment of sepsis and it is worth consideration as a treatment modality in clinical practice.

Previous studies have indicated that tanshinol may decrease the toxic effects of oxidative stresses, which in turn protects the cells from injury caused by oxidative stress and improves the ability of the body to inhibit toxicity and repair damaged cells $(14,17)$. In addition, sepsis is markedly associated with AQP-5, the water channel membrane protein, which has been established to serve pivotal roles in various human diseases (22-24). Consistent with previous studies, the present study demonstrated that the expression level of AQP-5 was decreased following infection $(23,25)$, indicating that AQP-5 is involved in the physiological changes caused by infection. Consistent results were identified in the present study with the mRNA and protein levels of AQP-5 being demonstrated to be significantly decreased in the rats belonging to the sepsis group when compared with rats of the ctrl and SO groups. Following treatment with tanshinol in a dose-dependent manner, the mRNA and protein levels of AQP-5 in rats with sepsis were increased, indicating that tanshinol may induce the expression of AQP-5. A previous study suggested that in sepsis, bacterial toxins cause the release of cytokines and the activation of mediator systems (29). TNF- $\alpha$ and IL-6 are two proinflammatory cytokines that may promote systemic inflammation (30). In the present study, the levels of TNF- $\alpha$ and IL- 6 in the sepsis model group were significantly increased compared with that of the control group, indicating the inflammation response was as a result of sepsis. However, when compared with the sepsis group, the levels of TNF- $\alpha$ and IL- 6 in the rats with sepsis were significantly decreased following treatment with tanshinol in a dose-dependent manner. Therefore, the results of the present study suggest that tanshinol may protect injured tissue and inhibit systemic inflammation by upregulating AQP-5 and downregulating proinflammatory cytokines including TNF- $\alpha$ and IL- 6 . Therefore, tanshinol may decrease the production of cytokines caused by sepsis in immune systems.

To fully explore the mechanism of the efficiency of tanshinol on sepsis, the present study demonstrated that tanshinol exhibits protective effects by upregulating AQP-5 and downregulating the proinflammatory cytokines that are associated with mitogen-activated protein kinase signaling pathway. P38, as a mitogen-activated protein kinase that is responsive to stress stimuli, serves a pivotal role in the response to infection $(31,32)$. The P38 signaling pathway may be activated by phosphorylation to form p-P38 following infection (32). Consequently, the results of the present study revealed that the level of p-P38 in the sepsis group was 
significantly increased compared with that in the ctrl and SO groups, indicating that the P38 signaling pathway was activated. Following treatment with tanshinol, the p-P38:P38 ratio of rats with sepsis was significantly decreased. These data suggested that sepsis may activate the P38 signaling pathway by phosphorylation, while downregulation of the proinflammatory cytokines by tanshinol may inhibit the activated P38 caused by sepsis. Taken together, tanshinol regulates the expression of AQP-5 in sepsis by downregulating the proinflammatory cytokines TNF- $\alpha$ and IL-6 through the P38 signaling pathway in sepsis.

In conclusion, the effect of tanshinol on the expression of $\mathrm{AQP}-5$ in the lung tissue of rats with sepsis was investigated through the establishment of a sepsis model by CLP. In the present study, an experimental model of sepsis was used to examine the effects of tanshinol to prevent sepsis-induced damage to lung tissue. Tanshinol increased the expression of AQP-5 in the lung tissue of rats with sepsis, potentially by inhibiting the expression of inflammatory factors and P38 phosphorylation. However, there are limitations in present study. On the one hand, the sample size of this study is relatively small, which may increase the experimental error. Furthermore, whether tanshinol can be used in clinical treatment of sepsis still requires experimental verification.

\section{Acknowledgements}

Not applicable.

\section{Funding}

The present study was supported by Projects of Traditional Medical Technology Development Program in Shandong province (grant no. 2015-417).

\section{Availability of data and materials}

All data generated or analyzed during this study are included in this published article.

\section{Authors' contributions}

JX, LY and LD designed the study. JX and LY analyzed and interpreted the data. JX, LY and LD wrote and revised the manuscript. All authors read and approved the final manuscript.

\section{Ethics approval and consent to participate}

All animal procedures performed in the present study were reviewed and approved Animal Ethics Committee of Qilu Hospital of Shandong University (Jinan, China).

\section{Patient consent for publication}

Not applicable.

\section{Competing interests}

The authors declare that they have no competing interests.

\section{References}

1. Martin GS: Sepsis, severe sepsis and septic shock: Changes in incidence, pathogens and outcomes. Expert Rev Anti Infect Ther 10: 701-706, 2012.

2. Angus DC and van der Poll T: Severe sepsis and septic shock. N Engl J Med 369: 840-851, 2013.

3. Dellinger RP, Levy MM, Rhodes A, Annane D, Gerlach H, Opal SM, Sevransky JE, Sprung CL, Douglas IS, Jaeschke R, et al: Surviving sepsis campaign: International guidelines for management of severe sepsis and septic shock, 2012. Intens Care Med 39: 165-228, 2013.

4. Levy MM, Fink MP, Marshall JC, Abraham E, Angus D, Cook D, Cohen J, Opal SM, Vincent JL and Ramsay G: SCCM/ESICM/ACCP/ATS/SIS: 2001 SCCM/ESICM/ACCP/ATS/SIS International Sepsis Definitions onference. Crit Care Med 31: 1250-1256, 2003.

5. Kaukonen KM, Bailey M, Suzuki S, Pilcher D and Bellomo R: Mortality related to severe sepsis and septic shock among critically ill patients in Australia and New Zealand, 2000-2012. JAMA 311: 1308-1316, 2014.

6. Bernard GR, Vincent JL, Laterre PF, LaRosa SP, Dhainaut JF, Lopez-Rodriguez A, Steingrub JS, Garber GE, Helterbrand JD, Ely EW, et al: Efficacy and safety of recombinant human activated protein C for severe sepsis. N Engl J Med 344: 699-709, 2001.

7. Rivers E, Nguyen B, Havstad S, Ressler J, Muzzin A, Knoblich B, Peterson E and Tomlanovich M; Early Goal-Directed Therapy Collaborative Group: Early goal-directed therapy in the treatment of severe sepsis and septic shock. N Engl J Med 345: 1368-1377, 2001.

8. Peake SL, Bailey M, Bellomo R, Cameron PA, Cross A, Delaney A, Finfer S, Higgins A, Jones DA, Myburgh JA, et al: Australasian resuscitation of sepsis evaluation (ARISE): A multi-centre, prospective, inception cohort study. Resuscitation 80: 811-818, 2009.

9. Wawrzeniak IC, Loss SH, Moraes MC, De La Vega FL and Victorino JA: Could a protocol based on early goal-directed therapy improve outcomes in patients with severe sepsis and septic shock in the intensive care unit setting? Indian J Crit Care Med 19: 159-165, 2015.

10. Zhang Y, Li X and Wang Z: Antioxidant activities of leaf extract of Salvia miltiorrhiza Bunge and related phenolic constituents. Food Chem Toxicol 48: 2656-2662, 2010.

11. Son B, Jun SY, Seo H, Youn H, Yang HJ, Kim W, Kim HK, Kang $\mathrm{C}$ and Youn B: Inhibitory effect of traditional oriental medicine-derived monoamine oxidase $\mathrm{B}$ inhibitor on radioresistance of non-small cell lung cancer. Sci Rep 6: 21986, 2016.

12. Guan Y, Wu XX, Duan JL, Yin Y, Guo C, Wei G, Wang YH, Zhu YR, Weng Y, Xi MM and Wen AD: Effects and mechanism of combination of rhein and danshensu in the treatment of chronic kidney disease. Am J Chin Med 43: 1381-1400, 2015.

13. Yu J, Wang L, Akinyi M, Li Y, Duan Z, Zhu Y and Fan G: Danshensu protects isolated heart against ischemia reperfusion injury through activation of Akt/ERK1/2/Nrf2 signaling. Int $\mathrm{J}$ Clin Exp Med 8: 14793-14804, 2015.

14. Yang Y, Su Y, Wang D, Chen Y, Wu T, Li G, Sun X and Cui L: Tanshinol attenuates the deleterious effects of oxidative stress on osteoblastic differentiation via Wnt/FoxO3a signaling. Oxid Med Cell Longev 2013: 351895, 2013.

15. Liu XH, Pan LL, Jia YL, Wu D, Xiong QH, Wang Y and Zhu YZ: A novel compound DSC suppresses lipopolysaccharide-induced inflammatory responses by inhibition of Akt/NF- $\mathrm{kB}$ signalling in macrophages. Eur J Pharmacol 708: 8-13, 2013.

16. Yang Y, Wang L, Wu Y, Su D, Wang N, Wang J, Shi C, Lv L and Zhang S: Tanshinol suppresses inflammatory factors in a rat model of vascular dementia and protects LPS-treated neurons via the MST1-FOXO3 signaling pathway. Brain Res 1646: 304-314, 2016.

17. Shioji I: Oxidative stress related diseases and biopyrrins. Rinsho Byori 53: 155-159, 2005 (In Japanese).

18. Kubota M, Hasegawa T, Nakakura T, Tanii H, Suzuki M and Tanaka S: Molecular and cellular characterization of a new aquaporin, AQP-x5, specifically expressed in the small granular glands of Xenopus skin. J Exp Biol 209: 3199-3208, 2006.

19. Delporte C, Bryla A and Perret J: Aquaporins in salivary glands: From basic research to clinical applications. Int J Mol Sci 17: pii: E166, 2016.

20. Verkman AS, Anderson MO and Papadopoulos MC: Aquaporins: Important but elusive drug targets. Nat Rev Drug Discov 13: 259-277, 2014. 
21. Shen Y, Wang Y, Chen Z, Wang D, Wang X, Jin M and Bai C: Role of aquaporin 5 in antigen-induced airway inflammation and mucous hyperproduction in mice. J Cell Mol Med 15: 1355-1363, 2011.

22. Gresz V, Horvath A, Gera I, Nielsen S and Zelles $T$ : Immunolocalization of AQP5 in resting and stimulated normal labial glands and in Sjögren's syndrome. Oral Dis 21: e114-e120, 2015.

23. Lin F, Pan LH, Ruan L, Qian W, Liang R, Ge WY and Huang B: Differential expression of HIF-1 $\alpha$, AQP-1, and VEGF under acute hypoxic conditions in the non-ventilated lung of a one-lung ventilation rat model. Life Sci 124: 50-55, 2015.

24. Han G, Ma L, Guo Y, Li L, Li D and Liu H: Hyperbaric oxygen therapy palliates lipopolysaccharide-induced acute lung injury in rats by upregulating AQP1 and AQP5 expression. Exp Lung Res 41: 444-449, 2015.

25. Zhang ZQ, Song YL, Chen ZH, Shen Y and Bai CX: Deletion of aquaporin 5 aggravates acute lung injury induced by Pseudomonas aeruginosa. J Trauma 71: 1305-1311, 2011.

26. Direito I, Madeira A, Brito MA and Soveral G: Aquaporin-5: From structure to function and dysfunction in cancer. Cell Mol Life Sci 73: 1623-1640, 2016.
27. Livak KJ and Schmittgen TD: Analysis of relative gene expression data using real-time quantitative PCR and the 2(-Delta Delta C(T)) method. Methods 25: 402-408, 2001.

28. Govindan S, Iwashyna TJ, Odden A, Flanders SA and Chopra V: Mobilization in severe sepsis: An integrative review. J Hosp Med 10: 54-59, 2015

29. Chen H, Xie K, Han H, Li Y, Liu L, Yang T and Yu Y: Molecular hydrogen protects mice against polymicrobial sepsis by ameliorating endothelial dysfunction via an $\mathrm{Nrf} 2 / \mathrm{HO}-1$ signaling pathway. Int Immunopharmacol 28: 643-654, 2015.

30. Yudkin JS, Kumari M, Humphries SE and Mohamed-Ali V: Inflammation, obesity, stress and coronary heart disease: Is interleukin-6 the link? Atherosclerosis 148: 209-214, 2000.

31. Khatri M and Sharma JM: Infectious bursal disease virus infection induces macrophage activation via p38 MAPK and NF-kappaB pathways. Virus Res 118: 70-77, 2006.

32. Rydkina E, Silverman DJ and Sahni SK: Activation of $\mathrm{p} 38$ stress-activated protein kinase during Rickettsia rickettsii infection of human endothelial cells: Role in the induction of chemokine response. Cell Microbiol 7: 1519-1530, 2005. 